\title{
Plane shearing waves of arbitrary form: Exact solutions of the Navier-Stokes equations
}

\author{
Nishant K. Singh ${ }^{1,2, a}$ and S. Sridhar ${ }^{3, b}$ \\ 1 Nordita, KTH Royal Institute of Technology and Stockholm University, Roslagstullsbacken 23, SE-10691 Stockholm, Sweden \\ 2 Max Planck Institute for Solar System Research, Justus-von-Liebig-Weg 3, D-37077 Göttingen, Germany \\ 3 Raman Research Institute, Sadashivanagar, Bangalore 560 080, India
}

Received: 20 July 2017

Published online: 28 September 2017

(C) The Author(s) 2017. This article is published with open access at Springerlink.com

\begin{abstract}
We present exact solutions of the incompressible Navier-Stokes equations in a background linear shear flow. The method of construction is based on Kelvin's investigations into linearized disturbances in an unbounded Couette flow. We obtain explicit formulae for all three components of a Kelvin mode in terms of elementary functions. We then prove that Kelvin modes with parallel (though time-dependent) wave vectors can be superposed to construct the most general plane transverse shearing wave. An explicit solution is given, with any specified initial orientation, profile and polarization structure, with either unbounded or shear-periodic boundary conditions.
\end{abstract}

\section{Introduction}

In 1986 Craik and Criminale [1] presented a class of exact solutions of the Navier-Stokes equations which were wavelike disturbances in background shear flows. Since then these solutions have proved extremely useful in the study of astrophysical and atmospheric fluid dynamics; a very useful collection of exact solutions can be found in [2]. The approach taken in [1] was a generalization of a century-old method invented by Kelvin [3] to study linearized perturbations of Couette flows; see also [4]. These shearing wave solutions, also referred to as Kelvin modes, have time-dependent wave vectors and amplitudes. This feature makes them extremely useful in local stability analysis [5, 6]. Although a single Kelvin mode is an exact solution of the full Navier-Stokes (NS) equations, it has been remarked [1] that until about 1965 there seems to be no evidence that this was so recognized; in fact, the first published mention is as late as 1983 [7]. Moreover, an explicit formula has been published [3,1] for only one of the three components of the disturbance.

In this paper we present exact solutions for all three components of the velocity field of a Kelvin mode, in closed form using only elementary mathematical functions. We identify a subset of these modes whose wave vectors - though time-dependent - remain parallel to each other for all time. These are used to synthesize the most general plane transverse shearing wave, which can have any specified initial orientation, profile and polarization structure, with either unbounded or shear-periodic boundary conditions.

Let $\left(\hat{\boldsymbol{e}}_{1}, \hat{\boldsymbol{e}}_{2}, \hat{\boldsymbol{e}}_{3}\right)$ be the unit basis vectors of a Cartesian coordinate system in the laboratory frame. Using notation $\boldsymbol{x}=\left(x_{1}, x_{2}, x_{3}\right)$ for the position vector and $t$ for time, we write the total fluid velocity as $\left(S x_{1} \hat{\boldsymbol{e}}_{2}+\boldsymbol{v}\right)$, where $S$ is the rate of shear parameter and $\boldsymbol{v}(\boldsymbol{x}, t)$ is the incompressible disturbance $(\boldsymbol{\nabla} \cdot \boldsymbol{v}=0)$, which obeys the NS equations

$$
\begin{aligned}
& \left(\partial_{t}+S x_{1} \partial_{2}\right) \boldsymbol{v}+S v_{1} \hat{\boldsymbol{e}}_{2}+(\boldsymbol{v} \cdot \boldsymbol{\nabla}) \boldsymbol{v}=-\boldsymbol{\nabla} p+\nu \nabla^{2} \boldsymbol{v}, \\
& \nabla^{2} p=-\boldsymbol{\nabla} \cdot[(\boldsymbol{v} \cdot \boldsymbol{\nabla}) \boldsymbol{v}]-2 S \partial_{2} v_{1} .
\end{aligned}
$$

\footnotetext{
a e-mail: singh@mps.mpg.de

b e-mail: ssridhar@rri.res.in
} 
We seek a solution in the form of a single Kelvin mode

$$
\begin{aligned}
& \boldsymbol{v}_{\boldsymbol{k}}(\boldsymbol{x}, t)=\operatorname{Re}\left\{\boldsymbol{A}(\boldsymbol{k}, t) \exp \left[\mathrm{i} \boldsymbol{k}^{\mathrm{sh}}(t) \cdot \boldsymbol{x}\right]\right\}, \\
& p_{\boldsymbol{k}}(\boldsymbol{x}, t)=\operatorname{Re}\left\{\psi(\boldsymbol{k}, t) \exp \left[\mathrm{i} \boldsymbol{k}^{\mathrm{sh}}(t) \cdot \boldsymbol{x}\right]\right\},
\end{aligned}
$$

where the time-dependent sheared wave vector, $\boldsymbol{k}^{\mathrm{sh}}(t)$, has components

$$
k_{1}^{\mathrm{sh}}=k_{1}-S t k_{2}, \quad k_{2}^{\mathrm{sh}}=k_{2}, \quad k_{3}^{\mathrm{sh}}=k_{3},
$$

with $\boldsymbol{k} \equiv\left(k_{1}, k_{2}, k_{3}\right)$ being a constant wave vector. Our task now is to determine the amplitudes $\boldsymbol{A}(\boldsymbol{k}, t)$. Incompressibility requires that $\boldsymbol{k}^{\mathrm{sh}}(t) \cdot \boldsymbol{A}(\boldsymbol{k}, t)=0$. Therefore, when eqs. (2) and (3) are substituted in eqs. (1), the nonlinear term, $(\boldsymbol{v} \cdot \boldsymbol{\nabla}) \boldsymbol{v}$ vanishes because

$$
(\boldsymbol{A} \cdot \boldsymbol{\nabla}) \exp \left[\mathrm{i} \boldsymbol{k}^{\mathrm{sh}}(t) \cdot \boldsymbol{x}\right]=\left(\mathrm{i} \boldsymbol{k}^{\mathrm{sh}}(t) \cdot \boldsymbol{A}\right) \exp \left[\mathrm{i} \boldsymbol{k}^{\mathrm{sh}}(t) \cdot \boldsymbol{x}\right]=0 .
$$

The pressure can be eliminated by using the second of eqs. (1): $\left|\boldsymbol{k}^{\mathrm{sh}}(t)\right|^{2} \psi=2 \mathrm{i} S k_{2} A_{1}$, where $\left|\boldsymbol{k}^{\mathrm{sh}}(t)\right|^{2}=\left[\left(k_{1}-S t k_{2}\right)^{2}+\right.$ $\left.k_{2}^{2}+k_{3}^{2}\right]$. Then $\boldsymbol{A}$ satisfies

$$
\partial_{t} \boldsymbol{A}+S A_{1} \hat{\boldsymbol{e}}_{2}=2 S\left(\frac{k_{2} \boldsymbol{k}^{\mathrm{sh}}(t)}{\left|\boldsymbol{k}^{\mathrm{sh}}(t)\right|^{2}}\right) A_{1}-\nu\left|\boldsymbol{k}^{\mathrm{sh}}(t)\right|^{2} \boldsymbol{A}
$$

We now obtain explicit solutions for $\boldsymbol{A}$. To do this, define a new amplitude variable, $\boldsymbol{a}(\boldsymbol{k}, t)$, by

$$
\boldsymbol{A}(\boldsymbol{k}, t)=\widetilde{G}_{\nu}(\boldsymbol{k}, t) \boldsymbol{a}(\boldsymbol{k}, t),
$$

where $\widetilde{G}_{\nu}(\boldsymbol{k}, t)$ is a Fourier-space viscous Green's function,

$$
\widetilde{G}_{\nu}(\boldsymbol{k}, t)=\exp \left[-\nu \int_{0}^{t} \mathrm{~d} s\left|\boldsymbol{k}^{\mathrm{sh}}(s)\right|^{2}\right]=\exp \left[-\nu\left(k^{2} t-S k_{1} k_{2} t^{2}+\frac{S^{2}}{3} k_{2}^{2} t^{3}\right)\right] .
$$

When eqs. (6) and (7) are substituted in eq. (5), we obtain the following equations for the three components of $\boldsymbol{a}(\boldsymbol{k}, t)$ :

$$
\begin{aligned}
\partial_{t} a_{1}-2 S\left[\frac{\left(k_{1}-S t k_{2}\right) k_{2}}{\left(k_{1}-S t k_{2}\right)^{2}+k_{2}^{2}+k_{3}^{2}}\right] a_{1} & =0, \\
\partial_{t} a_{2}-2 S\left[\frac{k_{2}^{2}}{\left(k_{1}-S t k_{2}\right)^{2}+k_{2}^{2}+k_{3}^{2}}-\frac{1}{2}\right] a_{1} & =0, \\
\partial_{t} a_{3}-2 S\left[\frac{k_{2} k_{3}}{\left(k_{1}-S t k_{2}\right)^{2}+k_{2}^{2}+k_{3}^{2}}\right] a_{1} & =0 .
\end{aligned}
$$

Equation (8) can be solved to get an explicit expression for $a_{1}(\boldsymbol{k}, t)$,

$$
a_{1}(\boldsymbol{k}, t)=\frac{k^{2}}{\left(k_{1}-S t k_{2}\right)^{2}+k_{2}^{2}+k_{3}^{2}} a_{1}(\boldsymbol{k}, 0),
$$

which is given in [3]. When this is substituted in eqs. (9) and (10), the latter can be integrated to obtain expressions for $a_{2}(\boldsymbol{k}, t)$ and $a_{3}(\boldsymbol{k}, t)$. However, neither Kelvin nor anyone else, to the best of our knowledge, have published explicit formulae for these two components ${ }^{1}$. Thus we were pleasantly surprised to find that $a_{2}(\boldsymbol{k}, t)$ and $a_{3}(\boldsymbol{k}, t)$ could be expressed entirely in terms of elementary functions:

$$
\begin{aligned}
a_{2}(\boldsymbol{k}, t)= & a_{2}(\boldsymbol{k}, 0)+\left\{\frac{k^{2} k_{3}^{2}}{k_{2}\left(k_{2}^{2}+k_{3}^{2}\right)^{3 / 2}}\left[\arctan \left(\frac{k_{1}-S t k_{2}}{\sqrt{k_{2}^{2}+k_{3}^{2}}}\right)-\arctan \left(\frac{k_{1}}{\sqrt{k_{2}^{2}+k_{3}^{2}}}\right)\right]\right. \\
& \left.-\frac{k^{2} k_{2}}{k_{2}^{2}+k_{3}^{2}}\left[\frac{k_{1}-S t k_{2}}{\left(k_{1}-S t k_{2}\right)^{2}+k_{2}^{2}+k_{3}^{2}}-\frac{k_{1}}{k^{2}}\right]\right\} a_{1}(\boldsymbol{k}, 0), \\
a_{3}(\boldsymbol{k}, t)= & a_{3}(\boldsymbol{k}, 0)-\left\{\frac{k^{2} k_{3}}{\left(k_{2}^{2}+k_{3}^{2}\right)^{3 / 2}}\left[\arctan \left(\frac{k_{1}-S t k_{2}}{\sqrt{k_{2}^{2}+k_{3}^{2}}}\right)-\arctan \left(\frac{k_{1}}{\sqrt{k_{2}^{2}+k_{3}^{2}}}\right)\right]\right. \\
& \left.+\frac{k^{2} k_{3}}{k_{2}^{2}+k_{3}^{2}}\left[\frac{k_{1}-S t k_{2}}{\left(k_{1}-S t k_{2}\right)^{2}+k_{2}^{2}+k_{3}^{2}}-\frac{k_{1}}{k^{2}}\right]\right\} a_{1}(\boldsymbol{k}, 0) .
\end{aligned}
$$

\footnotetext{
${ }^{1}$ Markus and Press [4] study perturbations of plane Couette flow using Kelvin waves. However, their analysis is limited to two-dimensional perturbations, whereas the shearing waves we consider here are fully three-dimensional.
} 

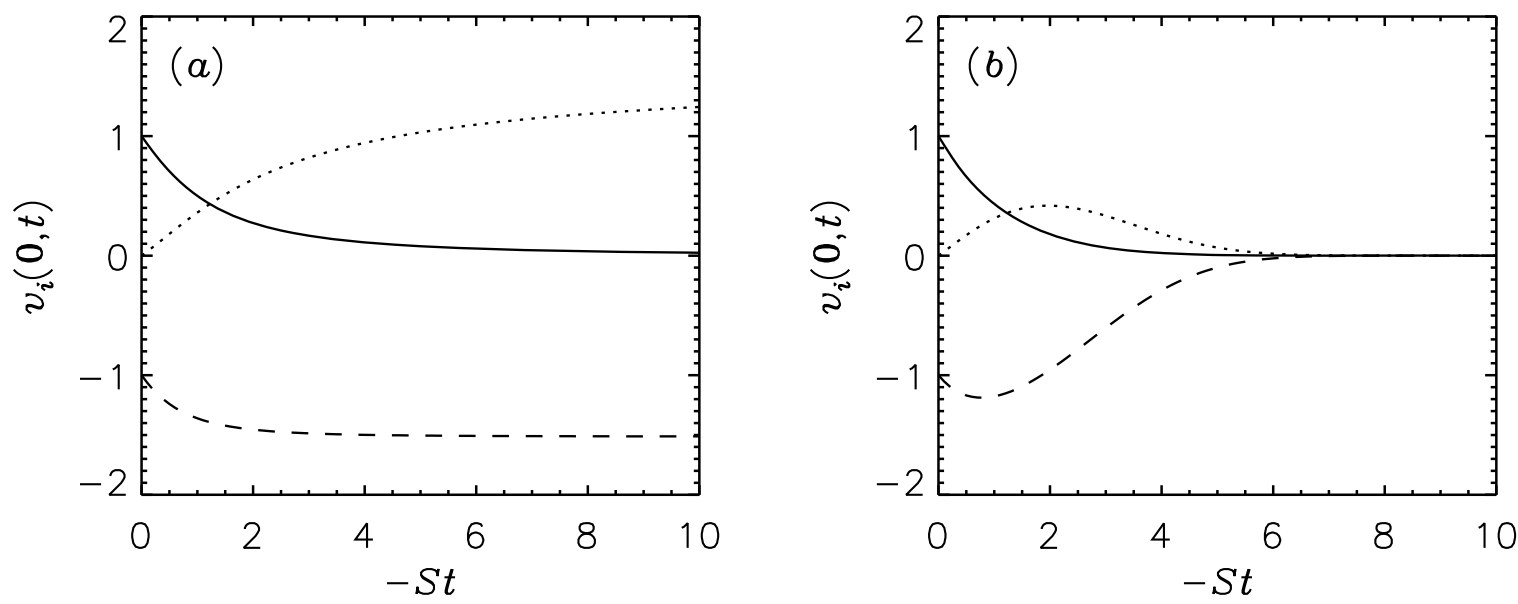

Fig. 1. Plots of the three components of the velocity field, measured at the origin, as functions of $S t$. We have chosen $\boldsymbol{k}=(1,1,1)$ and $\boldsymbol{a}(\boldsymbol{k}, 0)=(1,0,-1)$. The bold lines are for $v_{1}(\mathbf{0}, t)$, the dotted for $v_{2}(\mathbf{0}, t)$, and the dashed for $v_{3}(\mathbf{0}, t)$. Panel (a) is for the nonviscous case, $\nu=0$, so the velocity components are identical to the amplitudes, $\boldsymbol{a}(\boldsymbol{k}, t)$, of eqs. (11)-(13). Panel (b) corresponds to $\left(\nu k^{2} / S\right)=-0.1$, and all three components ultimately suffer viscous decay.

Incompressibility requires that $\boldsymbol{k}^{\mathrm{sh}}(t) \cdot \boldsymbol{a}(\boldsymbol{k}, t)=0$, which is guaranteed if the initial conditions are chosen such that $\boldsymbol{k} \cdot \boldsymbol{a}(\boldsymbol{k}, 0)=0$. From eqs. (11)-(13), we can see that, at late times, $a_{1}(\boldsymbol{k}, t) \rightarrow 0$, whereas both $a_{2}(\boldsymbol{k}, t)$ and $a_{3}(\boldsymbol{k}, t)$ saturate at non-zero values. This happens because the background flow shears out the $a_{1}$ component, and generates the $a_{2}$ and $a_{3}$ components.

When eqs. (6), (7), (11)-(13) are substituted in eq. (2), we obtain the full velocity field of a single Kelvin mode; it is readily verified that structure of the mode depends on the dimensionless variable, $S t$, and the dimensionless parameter, $\left(\nu k^{2} / S\right)$. The spatio-temporal behavior of these modes is briefly explored through figs. 1 and 2 . In order to understand its time variation, it is convenient to measure the velocity components at the origin, as is done in fig. 1 . Then, $\boldsymbol{v}(\mathbf{0}, t)=\widetilde{G}_{\nu}(\boldsymbol{k}, t) \operatorname{Re}\{\boldsymbol{a}(\boldsymbol{k}, t)\}$. Figure 1(a) corresponds to the case of zero viscosity, $\left(\nu k^{2} / S\right)=0$. In this case $\widetilde{G}_{\nu}=1$, and the plots give $\boldsymbol{v}(\mathbf{0}, t)=\operatorname{Re}\{\boldsymbol{a}(\boldsymbol{k}, t)\}$, where we can see the decay of $a_{1}$ and the saturation of $a_{2}$ and $a_{3}$ discussed above. In fig. 1(b), we have chosen $\left(\nu k^{2} / S\right)=-0.1$, so that all three components of $\boldsymbol{v}(\mathbf{0}, t)$ ultimately suffer viscous decay. It can be seen that, before this decay, there is transient amplification of $v_{2}$ and $v_{3}$, due to competition between shear and viscosity. For larger values of viscosity (not shown here), this transient amplification may be absent because the damping can overwhelm shear.

Until now we have considered an unbounded flow. However, in numerical simulations of the local dynamics of differentially rotating discs in astrophysical systems [8,9], it is customary to employ "shear-periodic" boundary conditions. Let us define sheared coordinates by

$$
x_{1}^{\mathrm{sh}}=x_{1}, \quad x_{2}^{\mathrm{sh}}=x_{2}-S t x_{1}, \quad x_{3}^{\mathrm{sh}}=x_{3} .
$$

These may be thought of as the Lagrangian coordinates of fluid elements that are carried along by the background shear flow. A function is said to be shear-periodic when it is a periodic function of $\left(x_{1}^{\mathrm{sh}}, x_{2}^{\mathrm{sh}}, x_{3}^{\mathrm{sh}}\right)$ with periodicities $\left(L_{1}, L_{2}, L_{3}\right)$, respectively. The phase of the function $\boldsymbol{v}_{\boldsymbol{k}}$ can be written as $\boldsymbol{k}^{\mathrm{sh}}(t) \cdot \boldsymbol{x}=\boldsymbol{k} \cdot \boldsymbol{x}^{\mathrm{sh}}$. Therefore, a shear-periodic Kelvin mode has wave vectors $\boldsymbol{k} \in\left(2 \pi m_{1} / L_{1}, 2 \pi m_{2} / L_{2}, 2 \pi m_{3} / L_{3}\right)$, where the $m_{i}$ take any integer values.

We now use the explicit expressions obtained for the Kelvin modes to construct the most general plane transverse shearing wave. Let us consider two Kelvin modes, $\boldsymbol{v}_{\boldsymbol{k}}(\boldsymbol{x}, t)$ and $\boldsymbol{v}_{\boldsymbol{k}^{\prime}}(\boldsymbol{x}, t)$ corresponding to wave vectors $\boldsymbol{k}$ and $\boldsymbol{k}^{\prime}$, which are parallel to each other but could differ in magnitudes. Using eqs. (3), we see that the corresponding sheared wave vectors, $\boldsymbol{k}^{\mathrm{sh}}(t)$ and $\boldsymbol{k}^{\prime} \mathrm{sh}(t)$, are also parallel to each other for all time. Incompressibility implies that $\boldsymbol{v}_{\boldsymbol{k}}(\boldsymbol{x}, t)$ and $\boldsymbol{v}_{\boldsymbol{k}^{\prime}}(\boldsymbol{x}, t)$ are perpendicular to $\boldsymbol{k}^{\mathrm{sh}}(t)$ and $\boldsymbol{k}^{\prime} \mathrm{sh}(t)$ for all time. So, if we superpose $\boldsymbol{v}_{\boldsymbol{k}}(\boldsymbol{x}, t)$ and $\boldsymbol{v}_{\boldsymbol{k}^{\prime}}(\boldsymbol{x}, t)$, the nonlinear term in the NS equations vanishes, because the superposed velocity field remains parallel to the wavefronts. Thus the superposition of an arbitrary number of Kelvin modes, all with wave vectors parallel to each other, is an exact solution of the NS equations.

Let us choose a unit vector $\hat{\boldsymbol{n}}=\left(n_{1}, n_{2}, n_{3}\right)$, and define the sheared (non-unit) vector $\boldsymbol{n}^{\text {sh }}(t)$ by

$$
n_{1}^{\mathrm{sh}}=\left(n_{1}-S t n_{2}\right), \quad n_{2}^{\mathrm{sh}}=n_{2}, \quad n_{3}^{\mathrm{sh}}=n_{3} .
$$



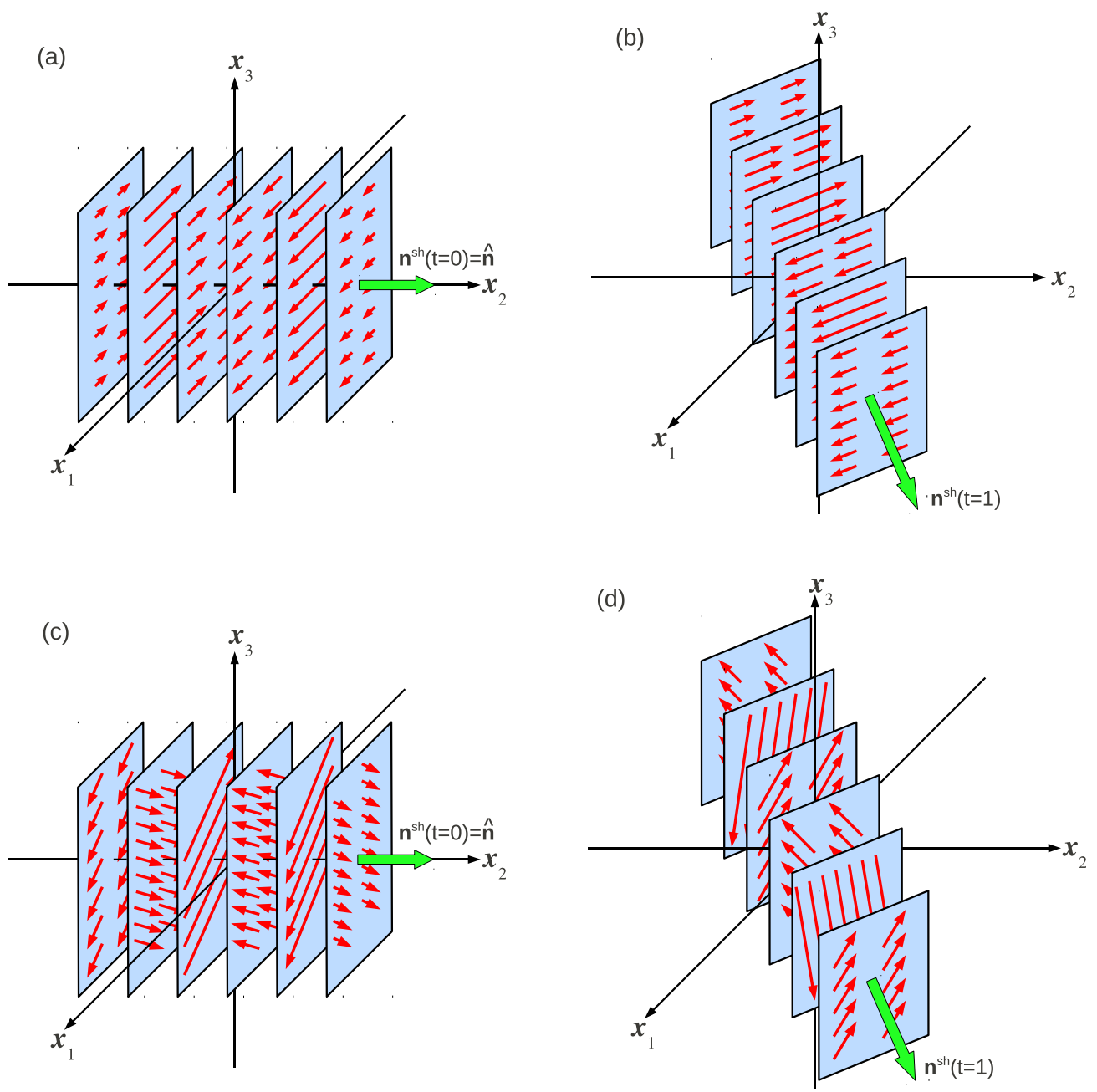

Fig. 2. Evolution of plane transverse shearing wavepackets. The polarization structure of the velocity field is indicated on some sections of the plane wavefronts. The parameters values used are $S=-1, \nu=1, W_{0}=1, \sigma=10$ and $k=1$. Panels (a) and (b): Linearly polarized $(h=0)$ wavepackets at times $t=0$ and $t=1$. Panels (c) and (d): Right circularly polarized $(h=1)$ at times $t=0$ and $t=1$.

Superposing all Kelvin modes with wave vectors $\boldsymbol{q}=q \hat{\boldsymbol{n}}$, where $-\infty<q<\infty$, we obtain an exact plane-wave solution of the NS equations with wavefronts perpendicular to $\boldsymbol{n}^{\mathrm{sh}}(t)$ :

$$
\begin{aligned}
v_{i}(\boldsymbol{x}, t)= & \int_{-\infty}^{\infty} \frac{\mathrm{d} q}{2 \pi} \widetilde{G}_{\nu}(q \hat{\boldsymbol{n}}, t) \widetilde{W}_{i}(q) \exp \left[\mathrm{i} q \boldsymbol{n}^{\mathrm{sh}}(t) \cdot \boldsymbol{x}\right] \\
& +\left[\frac{F_{i}\left(\boldsymbol{n}^{\mathrm{sh}}(t)\right)-F_{i}(\hat{\boldsymbol{n}})}{n_{2}^{2}+n_{3}^{2}}\right] \int_{-\infty}^{\infty} \frac{\mathrm{d} q}{2 \pi} \widetilde{G}_{\nu}(q \hat{\boldsymbol{n}}, t) \widetilde{W}_{1}(q) \exp \left[\mathrm{i} q \boldsymbol{n}^{\mathrm{sh}}(t) \cdot \boldsymbol{x}\right],
\end{aligned}
$$

where the dimensionless and scale-invariant functions, $F_{i}(\boldsymbol{Q})$, are defined by

$$
F_{i}(\boldsymbol{Q})=\frac{Q_{3}}{\sqrt{Q_{2}^{2}+Q_{3}^{2}}}\left[\frac{Q_{3}}{Q_{2}} \delta_{i 2}-\delta_{i 3}\right] \arctan \left(\frac{Q_{1}}{\sqrt{Q_{2}^{2}+Q_{3}^{2}}}\right)-\frac{Q_{1} Q_{i}}{Q^{2}} .
$$

For shear-periodic boundary conditions, the integral over $q$ in eq. (16) should be replaced by an appropriate sum. The $\widetilde{\boldsymbol{W}}(q)$ are Fourier-space initial conditions corresponding to the $\boldsymbol{a}(\boldsymbol{k}, 0)$ of eqs. (11)-(13), and must satisfy the incompressibility condition, $\hat{\boldsymbol{n}} \cdot \widetilde{\boldsymbol{W}}(q)=0$. They are determined by the initial profile and polarization stucture of the plane wave. At $t=0$, the wavefronts are perpendicular to $\hat{\boldsymbol{n}}$, so we write $\boldsymbol{v}(\boldsymbol{x}, 0)=\boldsymbol{W}(\hat{\boldsymbol{n}} \cdot \boldsymbol{x})$, where $\hat{\boldsymbol{n}} \cdot \boldsymbol{W}=0$. Note that the only constraint on the initial condition, $\boldsymbol{W}$, is that it is a vector field that is perpendicular everywhere to 
the unit vector $\hat{\boldsymbol{n}}$; otherwise it is a quite arbitrary function of its one argument. Thus, no restriction need be placed on the initial profile and polarization structure of the initial conditions. Given $\boldsymbol{W}(y)$, we can determine $\widetilde{\boldsymbol{W}}(q)=$ $\int_{-\infty}^{\infty} \mathrm{d} y \boldsymbol{W}(y) \exp [-\mathrm{i} q y]$, and use this in eq. (16) to calculate $\boldsymbol{v}(\boldsymbol{x}, t)$.

Equation (16) is a complete solution for a general plane shearing wave, expressed in terms of a Fourier integral. However, it is physically more transparent to rewrite the right side in terms of real-space quantities. To do this, we must introduce the real-space viscous Green's function, whose natural definition is with respect to the sheared coordinates $[10]$

$$
G_{\nu}\left(\boldsymbol{x}^{\mathrm{sh}}, t\right)=\int \frac{\mathrm{d} \boldsymbol{k}}{(2 \pi)^{3}} \widetilde{G}_{\nu}(\boldsymbol{k}, t) \exp \left[\mathrm{i} \boldsymbol{k} \cdot \boldsymbol{x}^{\mathrm{sh}}\right] .
$$

The properties of this function are discussed in $[11,10]$, where it is shown that it takes the form of a sheared heat kernel, which is an anisotropic Gaussian function of $\boldsymbol{x}^{\mathrm{sh}}$ with time-dependent coefficients; all the principal axes increase without bound and rotate against the direction of the background shear. Noting that $\boldsymbol{n}^{\operatorname{sh}}(t) \cdot \boldsymbol{x}=\hat{\boldsymbol{n}} \cdot \boldsymbol{x}^{\mathrm{sh}}$, we can write the general form of the plane shearing wave as

$$
v_{i}(\boldsymbol{x}, t)=\int \mathrm{d}^{3} \xi G_{\nu}(\boldsymbol{\xi}, t) W_{i}\left(\hat{\boldsymbol{n}} \cdot\left[\boldsymbol{x}^{\mathrm{sh}}(t)-\boldsymbol{\xi}\right]\right)+\left[\frac{F_{i}\left(\boldsymbol{n}^{\mathrm{sh}}(t)\right)-F_{i}(\hat{\boldsymbol{n}})}{n_{2}^{2}+n_{3}^{2}}\right] \int \mathrm{d}^{3} \xi G_{\nu}(\boldsymbol{\xi}, t) W_{1}\left(\hat{\boldsymbol{n}} \cdot\left[\boldsymbol{x}^{\mathrm{sh}}(t)-\boldsymbol{\xi}\right]\right) .
$$

As an illustrative example let us consider the following initial condition, corresponding to a polarized wavepacket with wave vector pointing along the $x_{2}$-axis: $\hat{\boldsymbol{n}}=\hat{\boldsymbol{e}}_{2}, W_{1}\left(x_{2}\right)=W_{0} \exp \left[-x_{2}^{2} / 2 \sigma^{2}\right] \sin k x_{2}, W_{2}=0, W_{3}\left(x_{2}\right)=$ $h W_{0} \exp \left[-x_{2}^{2} / 2 \sigma^{2}\right] \cos k x_{2}$, where $-1 \leq h \leq 1$. The wavepacket is linearly polarized when $h=0$, and right/left circularly polarized when $h= \pm 1$; other values of $h$ correspond to different degrees of elliptical polarizations. At a later time, the wave vector has components $n_{1}^{\text {sh }}=-S t, n_{2}^{\text {sh }}=1, n_{3}^{\text {sh }}=0$. Since both $W_{i}$ and $G_{\nu}(\boldsymbol{\xi}, t)$ are Gaussian functions, the integrals in eq. (19) can be performed analytically and $\boldsymbol{v}(\boldsymbol{x}, t)$ evaluated explicitly. We present the results graphically in fig. 2 for two cases, one linearly polarized and the other right circularly polarized. As the wavepackets are sheared, they undergo transient amplification due to the combined action of shear and viscosity, and at late times suffer viscous damping.

In conclusion, we have constructed exact solutions of the Navier-Stokes equation with a background linear shear flow. All three components of the velocity field of the Kelvin modes are given in closed form using only elementary mathematical functions. It is demonstrated that, when Kelvin modes with parallel wave vectors are superposed, they remain exact solutions. We give in explicit form the most general plane transverse shearing waves, with any specified initial orientation, profile and polarization structure, with either unbounded or shear-periodic boundary conditions. Of particular interest is the stability of our solutions; if they are stable then they might serve as local representations of disturbances in simulations of astrophysical flows.

Open access funding provided by Max Planck Society.

Open Access This is an open access article distributed under the terms of the Creative Commons Attribution License (http://creativecommons.org/licenses/by/4.0), which permits unrestricted use, distribution, and reproduction in any medium, provided the original work is properly cited.

\section{References}

1. A.D.D. Craik, W.O. Criminale, Proc. R. Soc. London A 406, 13 (1986).

2. P.G. Drazin, N. Riley, The Navier-Stokes equations: a classification of flows and exact solutions, in London Mathematical Society Lecture Note Series, Vol. 334 (Cambridge University Press, 2006).

3. W. Thomson (Lord Kelvin), Philos. Mag. 24, 188 (1887).

4. P.S. Marcus, W.H. Press, J. Fluid Mech. 79, 525 (1977).

5. A. Lifschitz, E. Hameiri, Phys. Fluids A 3, 2644 (1991)

6. B. Eckhardt, D. Yao, Chaos, Solitons Fractals 5, 2073 (1995).

7. K.K. Tung, J. Fluid Mech. 133, 443 (1983).

8. J. Binney, S. Tremaine, Galactic Dynamics, second edition (Princeton University Press, 2008).

9. S.A. Balbus, J.F. Hawley, Rev. Mod. Phys. 70, 1 (1998).

10. S. Sridhar, N.K. Singh, J. Fluid Mech. 664, 265 (2010).

11. F. Krause, K.-H. Rädler, Elektrodynamik der mittleren Felder in turbulenten leitenden Medien und Dynamotheorie, in Ergebnisse der Plasmaphysik und der Gaselektronik, Band 2 (Akademie, 1971) pp. 2-154. 